\title{
Increased aortic stiffness predicts future development and progression of peripheral neuropathy in patients with type 2 diabetes: the Rio de Janeiro Type 2 Diabetes Cohort Study
}

\author{
Claudia R. L. Cardoso ${ }^{1}$ - Camila B. M. Moran ${ }^{2} \cdot$ Fernanda S. Marinho ${ }^{2} \cdot$ \\ Marcel T. Ferreira ${ }^{1} \cdot$ Gil F. Salles ${ }^{1}$
}

Received: 3 March 2015 / Accepted: 19 May 2015 / Published online: 5 June 2015

(C) Springer-Verlag Berlin Heidelberg 2015

\begin{abstract}
Aims/hypothesis Diabetic peripheral neuropathy (DPN) is a chronic microvascular complication that is strongly associated with poor glycaemic control and also with a worse prognosis. We aimed to evaluate the predictors of the development and progression of DPN in a cohort of high-risk patients with type 2 diabetes.

Methods In a prospective study, 477 patients with type 2 diabetes were clinically assessed for the presence of DPN at baseline and after a median follow-up of 6.2 years (range 210 years). Clinical laboratory data were obtained at study entry and throughout the follow-up. Aortic stiffness was assessed by the carotid-femoral pulse wave velocity (cfPWV) at baseline. Multivariate Poisson regression analysis was used to examine independent predictors of the development/progression of DPN.

Results At baseline, 135 patients (28\%) had DPN, and during follow-up 97 patients (20\%) had either a new development or a worsening of DPN. Patients who showed a development or progression of DPN were taller and had a longer duration of diabetes, a greater prevalence of other microvascular complications and hypertension, greater aortic stiffness and poorer glycaemic control than patients who did not have new or progressive neuropathy. After adjustments for the baseline
\end{abstract}

Gil F. Salles

gilsalles@hucff.ufrj.br

1 Department of Internal Medicine, University Hospital Clementino Fraga Filho, School of Medicine, Universidade Federal do Rio de Janeiro, Rua Croton 72, Jacarepaguá, Rio de Janeiro 22750-240, Brazil

2 Department of Occupational Therapy, University Hospital Clementino Fraga Filho, School of Medicine, Universidade Federal do Rio de Janeiro, Rio de Janeiro, Brazil prevalence of DPN, the patient's age and sex, and the time interval between DPN assessments; an increased aortic stiffness (cf-PWV $>10 \mathrm{~m} / \mathrm{s}$ ) were predictive of new/progressive DPN (incidence rate ratio $2.04,95 \%$ CI $1.28,3.23 ; p=0.002$ ). Other independent predictors were the mean first-year $\mathrm{HbA}_{1 \mathrm{c}}$ level $(p=0.05)$, nephropathy ( $p=0.006)$, arterial hypertension ( $p=0.06)$ and height $(p=0.03)$.

Conclusions/interpretation Increased aortic stiffness at baseline predicts the future development or progression of peripheral neuropathy, independent of diabetic metabolic control, suggesting a physiopathological link between macrovascular and microvascular abnormalities in type 2 diabetes.

Keywords Aortic stiffness - Diabetic peripheral neuropathy · Glycaemic control $\cdot$ Predictors · Type 2 diabetes

$\begin{array}{ll}\text { Abbreviations } \\ \text { cf-PWV } & \text { Carotid-femoral pulse wave velocity } \\ \text { DBP } & \text { Diastolic BP } \\ \text { DPN } & \text { Diabetic peripheral neuropathy } \\ \text { eGFR } & \text { Estimated GFR } \\ \text { IRR } & \text { Incidence rate ratio } \\ \text { NDS } & \text { Neuropathy Disability Score } \\ \text { SBP } & \text { Systolic BP }\end{array}$

\section{Introduction}

Typical diabetic peripheral neuropathy (DPN) is defined as a 'symmetrical, length-dependent sensorimotor polyneuropathy attributable to metabolic and microvessel alterations as a result of chronic hyperglycaemia exposure and cardiovascular risk covariates' [1] and affects both large and small nerve fibres. Its pathogenesis involves interactions between vascular and 
metabolic factors [2]. Indeed, microcirculatory alterations similar to those observed in diabetic retinopathy and nephropathy are associated with pathological alterations in the nerves [3]. Most importantly, DPN is an important cause of disability, is associated with a higher mortality and reduced quality of life $[1,4,5]$ and imposes a tremendous cost on healthcare systems $[5,6]$.

Long-tem hyperglycaemia seems to be the most important risk factor for DPN; currently, besides improving glycaemic control, there is no other effective treatment to prevent the development or progression of DPN. However, neuropathy can develop despite intensive glucose control, suggesting that other risk factors are involved in the pathophysiology of DPN. Although intensive glucose control has been shown to prevent neuropathy in type 1 diabetes [7, 8], similar control seems to be less effective in type 2 diabetes [9-12]. These studies suggest that factors other than chronic hyperglycaemia, including metabolic factors such as dyslipidaemia, chronic inflammation and vascular risk factors, are probably interacting in the development of DPN [2, 7-12]. Thus, identifying other possibly modifiable factors for the development and progression of DPN could have potential future implications for treatment.

Central (aortic) arterial stiffness has been proposed as a measure of the burden of cumulative vascular risk factors [13]. Patients with type 2 diabetes show increased aortic stiffness [14], and associations between the presence of DPN and increased arterial stiffness have previously been demonstrated $[15,16]$. Nonetheless, longitudinal relationships between increased aortic stiffness and the development/progression of DPN have not yet been investigated. Hence, we aimed to explore the independent predictors of the development/ progression of DPN in a cohort of high-risk patients with type 2 diabetes, paying particular attention to the prognostic importance of increased aortic stiffness, assessed by its gold standard method, the carotid-femoral pulse wave velocity (cfPWV) [17].

\section{Methods}

This was a prospective study, nested within the Rio de Janeiro Type 2 Diabetes Cohort Study, that involved 477 patients with type 2 diabetes who were enrolled between 2004 and 2008 and re-evaluated for the diagnosis of peripheral neuropathy between 2013 and 2014 in the diabetes outpatient clinic of our tertiary-care University Hospital. All participants gave written informed consent, and the local Ethics Committee had previously approved the study protocol.

The characteristics of this cohort, the baseline procedures and the diagnostic definitions have been detailed elsewhere $[15,18-20]$. In brief, the inclusion criteria were all adults with type 2 diabetes who were aged up to 80 years and had either any microvascular (retinopathy, nephropathy or neuropathy) or macrovascular (coronary, cerebrovascular or peripheral artery disease) complication, or at least two other modifiable cardiovascular risk factors. The exclusion criteria were morbid obesity (BMI $\geq 40 \mathrm{~kg} / \mathrm{m}^{2}$ ), advanced renal failure (serum creatinine $>180 \mu \mathrm{mol} / 1$ or an estimated GFR [eGFR] $<30 \mathrm{ml} /$ $\min / 1.73 \mathrm{~m}^{2}$ ) or the presence of any serious concomitant disease limiting life expectancy. All the patients were submitted to a standard baseline protocol that included a thorough clinical examination, including a complete evaluation for peripheral neuropathy and a history of alcohol intake, a laboratory evaluation and an assessment of aortic stiffness in terms of cfPWV measurement.

The diagnostic criteria for chronic complications of diabetes have previously been detailed [15, 18-20]. Briefly, CHD was diagnosed by clinical electrocardiographic criteria or by positive ischaemic stress tests. Cerebrovascular disease was diagnosed by a history and physical examination, and peripheral arterial disease by an ankle-brachial index $<0$.9. Diabetic retinopathy was evaluated by an ophthalmologist. The diagnosis of nephropathy (incipient or overt) needed at least two findings of albuminuria $\geq 30 \mathrm{mg} / 24 \mathrm{~h}$ or a confirmed reduction in eGFR $\left(<60 \mathrm{ml} / \mathrm{min} / 1.73 \mathrm{~m}^{2}\right.$; or serum creatinine $>130 \mu \mathrm{mol} / \mathrm{l})$. Clinic BP was measured three times using a digital oscillometric BP monitor (HEM-907XL; Omron Healthcare, Kyoto, Japan) with a suitably sized cuff on two occasions 2 weeks apart at study entry. The first measurement at each visit was discarded, and the BP considered was the mean of the last two readings from each visit. Arterial hypertension was diagnosed if the mean systolic BP (SBP) was $\geq 130 \mathrm{mmHg}$ or the mean diastolic BP (DBP) was $\geq 80 \mathrm{mmHg}$, or if antihypertensive drugs had been prescribed.

Laboratory evaluation included fasting glycaemia, $\mathrm{HbA}_{1 \mathrm{c}}$, serum creatinine, lipids and vitamin $\mathrm{B}_{12}$. Those patients (3.4\%) with low serum vitamin $B_{12}$ levels $(<150 \mathrm{pmol} / \mathrm{l})$ were started on monthly intramuscular vitamin $\mathrm{B}_{12}$ administration. Albuminuria and proteinuria were evaluated in two nonconsecutive sterile $24 \mathrm{~h}$ urine collections. Aortic stiffness was evaluated by cf-PWV, using the foot-to-foot velocity method with Complior equipment and software (Artech Medical, Paris, France), as previously described [14, 17]. The direct carotid-femoral distance was corrected by a factor of 0.8 , and a cf-PWV $>10 \mathrm{~m} / \mathrm{s}$ was considered to represent increased aortic stiffness, as recommended [21]. Patients with aortoiliac occlusive disease were excluded because of its known attenuating effect on PWV measurements. The patients were followed up regularly at least three or four times a year until December 2014. All patients had at least two to four annual $\mathrm{HbA}_{1 \mathrm{c}}$ and clinic $\mathrm{BP}$ measurements.

Assessment of peripheral neuropathy Neuropathic symptoms were evaluated by a standard validated questionnaire [22], which allocates between 0 and 9 points to specific symptoms. Patients were asked about pain or discomfort in their 
legs: if the patient described burning, numbness or tingling, a score of 2 was assigned; fatigue, cramping or aching scored 1. The presence of symptoms in the feet was assigned a score of 2 , in the calves a score of 1 and elsewhere a score of 0 . The exacerbation of symptoms only at night scored 2 vs 1 for both day and night and 0 for daytime only. A score of 1 was given if the symptoms had ever woken the patient. The patients were asked if any manoeuvre could reduce their symptoms: walking was assigned a score of 2, standing was 1 and sitting or lying down was 0 [22]. Patients were classified as having no specific symptoms ( $0-2$ points) or mild (3-4), moderate (5-6) or severe (7-9 points) symptoms.

The clinical signs of peripheral neuropathy were assessed by the Neuropathy Disability Score (NDS) [22] and by sensation in the feet tested with a Semmes-Weinstein $10 \mathrm{~g} / 5.07$ monofilament. The NDS combines examination of the ankle reflex with testing for vibration (with $128 \mathrm{~Hz}$ tuning fork), pinprick and temperature (with a cold tuning fork) sensations on both big toes, and gives a total score ranging from 0 (no signs) to 10 points. The sensory evaluations score either 0 (present) or 1 (reduced/absent), whereas the ankle reflex scores 0 (normal), 1 (present with reinforcement) or 2 (absent) [22]. Patients were classified as having mild neuropathic signs (3-5 points), moderate signs (6-8) or severe signs (9-10). In addition, sensation over the feet was tested with the Semmes-Weinstein monofilament on four plantar sites for each foot (the big toe and first, third and fifth metatarsal heads); if at least two insensitive regions were found, this was considered abnormal. All the examinations were repeated twice by the same independent observer, and in cases of disagreement a third examination was performed. The minimum criteria for the diagnosis of peripheral neuropathy were the presence of moderate symptoms (regardless of the presence of signs) or the presence of mild symptoms with moderate neuropathic signs. Hence, mild symptoms with or without mild signs were not considered adequate for establishing a diagnosis of peripheral neuropathy.

Patients were evaluated for neuropathy at study entry and after a median time interval of 6.2 years. In those with peripheral neuropathy at baseline, the criteria for defining the progression of the neuropathy were as follows: an increase in the neuropathic symptom score from mild to moderate or from moderate to severe; an increase in the NDS from mild or moderate to severe; a doubling in the number of insensitive areas on monofilament examination (for those with at least two insensitive areas at the first assessment); and the occurrence of classic non-traumatic diabetic foot ulceration or toe amputation.

Statistical analysis Continuous data were described as means (SD) or as medians (interquartile range). The primary endpoint was the new development of established peripheral neuropathy or the progression of a pre-existent neuropathy, according to the criteria detailed above. Patients with and without a development/progression of peripheral neuropathy were compared by unpaired $t$ test (for continuous normal variables), Mann-Whitney test (for continuous asymmetrical variables) and $\chi^{2}$ test (for categorical variables). Independent associations between aortic stiffness (analysed as both continuous and categorical cf-PWV) and the development/ progression of DPN were evaluated by multivariate Poisson (loglinear) regression analyses with increasing levels of statistical adjustment. In addition, the independent predictors of the occurrence of the primary endpoint were examined by multivariate Poisson regression.

Candidate variables to enter the multivariate regression models were the following: age, sex, BMI, height, duration of diabetes, smoking status, physical activity, dyslipidaemia, arterial hypertension, diabetic treatment (metformin and insulin), antihypertensive treatment (number and classes of drugs), mean first-year SBP, each macrovascular (coronary and cerebrovascular disease) and each microvascular (retinopathy, nephropathy and neuropathy) diabetic complication at baseline, $\mathrm{HbA}_{1 \mathrm{c}}$ level and increased aortic stiffness. Regardless of their significance, age, sex, the presence of peripheral neuropathy at baseline and the time interval between DPN assessments were forced into all the models. A forward selection procedure was used to select the independent predictors with a $\mathrm{p}$ value $<0.10$ as the criterion to enter and remain in the models. $\mathrm{HbA}_{1 \mathrm{c}}$ measurements at baseline and as mean levels at the first and second years of follow-up were evaluated in separate models adjusted for the same covariates as the original predictive model. Separate analyses were also performed for development of new DPN (i.e. including only patients without DPN at baseline) and progression of DPN (i.e. including only patients with DPN at baseline).

The Poisson regression results were presented as incidence rate ratios (IRR), calculated as the exponential of the estimated regression coefficients [23], with their respective 95\% CIs, and a two-tailed $\mathrm{p}$ value $<0.05$ was considered significant. Statistics were performed using SPSS version 19.0 (SPSS Inc, Chicago, IL, USA).

\section{Results}

At baseline, 135 patients (28\%) had DPN. After a median time interval of 6 years (interquartile range 5-7 years, total range 2-10 years, $), 97$ patients $(20 \%)$ showed either newly developed (50 patients) or worsened (47 patients) DPN. Of the 47 patients with progressive DPN, 17 developed non-traumatic foot ulcers (classic diabetic foot) or had toe/forefoot amputations, 12 at least doubled the number of insensitive points on monofilament examination, and 18 changed their neuropathy symptom score from mild to moderate or from moderate to 
Table 1 Characteristics of all diabetic patients divided according to the development or progression of peripheral neuropathy during follow-up

\begin{tabular}{|c|c|c|c|c|}
\hline Variable & $\begin{array}{l}\text { All patients } \\
(n=477)\end{array}$ & $\begin{array}{l}\text { Patients without development/ } \\
\text { progression of DPN }(n=380)\end{array}$ & $\begin{array}{l}\text { Patients with development / } \\
\text { progression of DPN }(n=97)\end{array}$ & $p$ value \\
\hline Age (years) & $59.3(9.0)$ & $59.3(8.9)$ & $59.9(10.4)$ & 0.57 \\
\hline Male $(\%)$ & 36.3 & 35.1 & 41.6 & 0.25 \\
\hline Height (m) & $1.60(0.10)$ & $1.59(0.09)$ & $1.63(0.11)$ & 0.014 \\
\hline BMI $\left(\mathrm{kg} / \mathrm{m}^{2}\right)$ & $29.8(4.7)$ & $29.7(4.8)$ & $30.2(4.4)$ & 0.36 \\
\hline Smoking, current/past (\%) & 43.7 & 44.7 & 39.3 & 0.37 \\
\hline Alcohol intake, current $(\%)$ & 12.4 & 12.1 & 13.3 & 0.72 \\
\hline Physical activity (\%) & 25.2 & 26.0 & 21.3 & 0.36 \\
\hline Duration of diabetes (years) & $8(3-15)$ & $7(3-15)$ & $11(7-20)$ & $<0.001$ \\
\hline \multicolumn{5}{|l|}{ Chronic diabetic complications (\%) } \\
\hline Cerebrovascular disease & 7.1 & 6.9 & 8.2 & 0.66 \\
\hline Coronary artery disease & 15.1 & 14.7 & 16.9 & 0.61 \\
\hline Retinopathy & 32.0 & 26.6 & 51.5 & $<0.001$ \\
\hline Nephropathy & 29.5 & 23.4 & 56.2 & $<0.001$ \\
\hline Peripheral neuropathy & 28.4 & 21.2 & 56.2 & $<0.001$ \\
\hline Cardiovascular autonomic neuropathy & 20.8 & 18.0 & 31.8 & 0.007 \\
\hline \multicolumn{5}{|l|}{ Diabetes treatment $(\%)$} \\
\hline Metformin & 89.0 & 91.2 & 84.3 & 0.064 \\
\hline Sulfonylureas & 43.7 & 43.2 & 46.1 & 0.62 \\
\hline Insulin & 47.9 & 44.6 & 60.8 & 0.004 \\
\hline Arterial hypertension (\%) & 85.2 & 83.1 & 95.5 & 0.003 \\
\hline Number of antihypertensive drugs & $3(1-3)$ & $2(1-3)$ & $3(2-3)$ & 0.021 \\
\hline ACE inhibitors/AR blockers (\%) & 82.8 & 81.6 & 87.2 & 0.22 \\
\hline Diuretics (\%) & 65.9 & 62.4 & 79.8 & 0.001 \\
\hline Calcium channel blockers (\%) & 31.0 & 30.3 & 34.0 & 0.53 \\
\hline Beta-blockers $(\%)$ & 48.3 & 46.5 & 55.3 & 0.13 \\
\hline Baseline clinic SBP (mmHg) & $147(24)$ & $146(24)$ & $152(26)$ & 0.032 \\
\hline Mean first-year SBP (mmHg) & $140(19)$ & $139(18)$ & $146(20)$ & 0.003 \\
\hline Mean second-year SBP (mmHg) & $140(19)$ & $139(18)$ & $144(20)$ & 0.026 \\
\hline Dyslipidaemia (\%) & 88.0 & 88.1 & 87.6 & 0.89 \\
\hline Statin use $(\%)$ & 76.4 & 77.0 & 75.3 & 0.73 \\
\hline \multicolumn{5}{|l|}{ Laboratory variables } \\
\hline Fasting glucose (mmol/l) & $8.6(3.5)$ & $8.7(3.6)$ & $9.5(4.2)$ & 0.093 \\
\hline Baseline $\mathrm{HbA}_{1 \mathrm{c}}(\%)$ & $8.0(1.9)$ & $7.9(1.9)$ & $8.5(2.1)$ & 0.012 \\
\hline Baseline $\mathrm{HbA}_{1 \mathrm{c}}(\mathrm{mmol} / \mathrm{mol})$ & $64(21)$ & $63(21)$ & $69(23)$ & \\
\hline Mean first-year $\mathrm{HbA}_{1 \mathrm{c}}(\%)$ & $7.7(1.5)$ & $7.6(1.4)$ & $8.2(1.7)$ & 0.004 \\
\hline Mean first-year $\mathrm{HbA}_{1 \mathrm{c}}(\mathrm{mmol} / \mathrm{mol})$ & $61(16)$ & $60(15)$ & $66(19)$ & \\
\hline Mean second-year $\mathrm{HbA}_{1 \mathrm{c}}(\%)$ & $7.8(1.6)$ & $7.6(1.5)$ & $8.2(1.7)$ & 0.007 \\
\hline Mean second-year $\mathrm{HbA}_{1 \mathrm{c}}(\mathrm{mmol} / \mathrm{mol})$ & $62(18)$ & $60(16)$ & $66(19)$ & \\
\hline Triacylglycerol (mmol/l) & $1.61(1.13-2.44)$ & $1.59(1.13-2.37)$ & $1.67(1.13-2.62)$ & 0.64 \\
\hline HDL-cholesterol (mmol/l) & $1.09(0.29)$ & $1.10(0.30)$ & $1.05(0.25)$ & 0.13 \\
\hline LDL-cholesterol (mmol/l) & $3.02(0.99)$ & $3.05(0.98)$ & $2.92(1.00)$ & 0.24 \\
\hline Vitamin $B_{12}(\mathrm{pmol} / \mathrm{l})$ & $317(165)$ & 309 (164) & $362(217)$ & 0.29 \\
\hline Glomerular filtration rate $\left(\mathrm{ml} / \mathrm{min} / 1.73 \mathrm{~m}^{2}\right)$ & $94(32)$ & $95(31)$ & $89(35)$ & $<0.001$ \\
\hline Albuminuria (mg/24 h) & $13(7-38)$ & $12(7-26)$ & $36(12-162)$ & $<0.001$ \\
\hline Aortic stiffness (cf-PWV, m/s) & $9.2(1.9)$ & $8.9(1.8)$ & $10.1(2.1)$ & $<0.001$ \\
\hline Increased aortic stiffness (cf-PWV $>10 \mathrm{~m} / \mathrm{s}, \%$ ) & 25.0 & 18.9 & 48.9 & $<0.001$ \\
\hline
\end{tabular}

Values are proportions, and means (SD) or medians (interquartile range)

$\mathrm{AR}$, angiotensin II receptor 
Table 2 Independent associations between aortic stiffness and the development or progression of DPN after increasing adjustments on Poisson regression analysis

\begin{tabular}{|c|c|c|c|c|}
\hline Aortic stiffness variables & $\begin{array}{l}\text { Model } 1 \\
\text { IRR }(95 \% \mathrm{CI})\end{array}$ & $\begin{array}{l}\text { Model } 2 \\
\text { IRR }(95 \% \text { CI) }\end{array}$ & $\begin{array}{l}\text { Model } 3 \\
\text { IRR }(95 \% \mathrm{CI})\end{array}$ & $\begin{array}{l}\text { Model } 4 \\
\text { IRR }(95 \% \text { CI) }\end{array}$ \\
\hline Continuous cf-PWV ( $1 \mathrm{~m} / \mathrm{s}$ increment $)$ & $1.20(1.12,1.29)^{* * *}$ & $1.18(1.10,1.27)^{* * *}$ & $1.15(1.07,1.24)^{* * *}$ & $1.11(1.01,1.22)^{*}$ \\
\hline Increased cf-PWV (>10 m/s) & $3.12(2.00,4.76)^{* * *}$ & $2.50(1.87,3.33)^{* * *}$ & $2.38(1.51,3.70)^{* * *}$ & $1.96(1.18,3.23)^{* *}$ \\
\hline
\end{tabular}

Model 1 is the crude unadjusted analysis

Model 2 is adjusted for age and sex

Model 3 is further adjusted for prevalent peripheral neuropathy and the time interval between neuropathy assessments

Model 4 is adjusted for age, sex, BMI, height, duration of diabetes, smoking status, alcohol intake, physical activity, dyslipidaemia, arterial hypertension, diabetic treatment (metformin and insulin), antihypertensive treatment (number and classes of drugs), mean first-year SBP, each macrovascular (coronary and cerebrovascular disease) and microvascular (retinopathy, nephropathy and neuropathy) diabetic complication at baseline, mean first-year $\mathrm{HbA}_{1 \mathrm{c}}$ and the time interval between neuropathy assessments

${ }^{*} p<0.05, * * p<0.01, * * * p<0.001$

severe, and/or recorded an increase in NDS from mild/moderate to severe.

Table 1 outlines the characteristics of all the patients and of those with and without a development/progression of DPN. Patients who had newly developed or progressive peripheral neuropathy were taller and had a longer duration of diabetes, a greater prevalence of other microvascular complications and greater aortic stiffness than patients who did not show a development or progression of neuropathy. They also had a greater prevalence of hypertension and higher SBP at baseline and during follow-up, although they used more antihypertensive medications. In addition, these patients showed poorer glycaemic control at baseline and during follow-up, although they used insulin more frequently than those without a development/progression of DPN. There were no differences in the proportion of patients who were currently drinkers of alcohol, or in serum vitamin $\mathrm{B}_{12}$ levels, between those with and without a development/progression of DPN.
Table 2 presents the results of the independent associations between aortic stiffness and the development/progression of DPN from Poisson regression with several increasing adjustments. Both continuous and categorical cf-PWV predicted the development/progression of DPN in all models. An increased aortic stiffness $(>10 \mathrm{~m} / \mathrm{s})$ at baseline doubled the incidence rate of developing/worsening DPN, whereas each $1 \mathrm{~m} / \mathrm{s}$ increment in cf-PWV increased by $11 \%$ the incidence rate of the development/progression of DPN during follow-up in the most adjusted models.

Table 3 shows the other independent predictors of the development/progression of DPN. These were the presence of nephropathy, arterial hypertension (but not clinic SBP levels), height and poor glycaemic control during the first year of follow-up. Similar results were seen after entering the albuminuria status ( $<30 \mathrm{vs} \geq 30 \mathrm{mg} / 24 \mathrm{~h}$ ) and eGFR separately instead of the diagnosis of diabetic nephropathy: the presence of microalbuminuria was associated with an $80 \%$ increased
Table 3 Results of multivariate Poisson regression analysis of the independent predictors of the development or progression of peripheral neuropathy in patients with type 2 diabetes after a median follow-up of 6.2 years

\begin{tabular}{lccc}
\hline Independent predictors & IRR & $95 \%$ CI & $p$ value \\
\hline Increased aortic stiffness (cf-PWV $>10 \mathrm{~m} / \mathrm{s})$ & 2.04 & $1.28,3.23$ & 0.002 \\
Nephropathy & 1.89 & $1.20,3.03$ & 0.006 \\
Height $(10 \mathrm{~cm}$ increment) & 1.39 & $1.03,1.87$ & 0.03 \\
Mean first-year $\mathrm{HbA}_{1 \mathrm{c}}(1 \%$ increment) & 1.13 & $1.00,1.29$ & 0.05 \\
Arterial hypertension & 2.38 & $0.95,5.88$ & 0.06 \\
Female sex & 1.59 & $0.87,2.86$ & 0.13 \\
Age (10 year increment) & 1.06 & $0.80,1.39$ & 0.70 \\
Peripheral neuropathy at baseline & 2.00 & $1.52,3.13$ & 0.003 \\
Time interval between DPN assessments (months) & 1.00 & $0.99,1.01$ & 0.51 \\
\hline
\end{tabular}

Candidate variables to enter the model were: age, sex, BMI, height, duration of diabetes, smoking status, alcohol intake, physical activity, dyslipidaemia, arterial hypertension, diabetic treatment (metformin and insulin), antihypertensive treatment (number and classes of drugs), mean first-year SBP, each macrovascular (coronary and cerebrovascular disease) and microvascular (retinopathy, nephropathy and neuropathy) diabetic complication at baseline, mean first-year $\mathrm{HbA}_{1 \mathrm{c}}$ and increased aortic stiffness. Regardless of their significance, age, sex, the presence of peripheral neuropathy at baseline and the time interval between DPN assessments were forced into the model 
risk of the development/progression of DPN (IRR 1.79, 95\% CI 1.14, 2.86; $p=0.012$ ), whereas the eGFR was not associated with the development/progression of DPN $(p=0.29)$.The presence of diabetic retinopathy at baseline was not associated with a development/worsening of DPN regardless of entering or not entering nephropathy into the multivariate models. In terms of glycaemic control, only the mean $\mathrm{HbA}_{1 \mathrm{c}}$ during the first year of follow-up, but not the baseline $\mathrm{HbA}_{1 \mathrm{c}}$ or its mean level for the second year, predicted the development/ progression of peripheral neuropathy.

In separate analyses (Table 4), increased aortic stiffness and the presence of nephropathy were predictors of both the development and the progression of DPN (albeit being of borderline significance for the progression of DPN). However, the $95 \%$ CIs of the IRRs for height, mean $\mathrm{HbA}_{1 \mathrm{c}}$ and arterial hypertension were too wide to allow a meaningful interpretation of these results because of the lower statistical power of these separate analyses.

\section{Discussion}

This prospective study over a 6.2 year median follow-up has three main findings. First, it confirms the role of good glycaemic control in preventing the development/ progression of DPN for high-risk, mostly elderly patients with long-standing type 2 diabetes. Second, it demonstrates for the first time the importance of increased aortic stiffness as a predictor of the future development/progression of DPN. Finally, it shows the contribution of other covariates such as vascular risk factors (hypertension), anthropometric factors (height) and the presence of other microvascular complications (nephropathy or abnormal albuminuria) to helping in the identification of individuals at high risk of the development/ progression of DPN. These findings have potential clinical implications: beyond achieving optimal glycaemic control, the control of blood pressure, reduction in albuminuria and attenuation of aortic stiffness may contribute to preventing
DPN. This speculation, however, clearly needs to be addressed in future prospective interventional trials.

The association of increased aortic stiffness at baseline with the future development/progression of DPN is a new finding. We $[15]$ and others $[16,24]$ have previously reported that aortic stiffness was associated with DPN in cross-sectional analyses, but none of these had evaluated longitudinal temporal relationships. Although observational cohort studies did not allow causal inferences, this finding suggests a common physiopathological link between macrovascular and microvascular abnormalities in type 2 diabetes. Increased aortic stiffness may simply be a surrogate measure of the cumulative burden of vascular risk factors on the vessel wall over time, as previously proposed $[13,25]$, reflecting the well-accepted interaction between metabolic and vascular factors in the pathogenesis of DPN $[1,2]$. Increased aortic stiffness can directly damage the microcirculation, including the vasa nervorum, by increasing the transmission of wider, harmful pulsatile pressure waves as a result of the loss of the normal aortic buffering function [13,14]. Alternatively, both DPN and aortic stiffness may share common determinants, such as oxidative stress, endothelial dysfunction, the formation of advanced glycation end-products and vessel wall inflammation. Interestingly, we recently reported [26] that unstiffening of the aorta can be achieved in patients with type 2 diabetes by optimal glycaemic and blood pressure control, which were also associated with the prevention of DPN.

Although the cornerstone role of optimal glycaemic control in preventing the development/progression of DPN in patients with type 1 diabetes is undisputed [1, 7, 8, 27], its importance in type 2 diabetes is more debatable. In addition, even for patients with type 1 diabetes, current strategies for optimising glycaemic control are insufficient to fully prevent or delay the development of DPN seen on long-term follow-up [28]. The Action to Control Cardiovascular Risk in Diabetes (ACCORD) Trial [9], although it reported a lack of benefit of intensive glucose control for the primary microvascular endpoints (a composite of advanced renal, retinal and neuropathy endpoints), did find a benefit in terms of preventing most

Table 4 Separate Poisson regression analyses for the predictors of the development and progression of DPN during follow-up

\begin{tabular}{lll}
\hline Independent predictors & $\begin{array}{l}\text { New DPN development } \\
(342 \text { patients, 50 events) } \\
\text { IRR }(95 \% \mathrm{CI})\end{array}$ & $\begin{array}{l}\text { DPN progression } \\
(135 \text { patients, 47 events) } \\
\text { IRR (95\% CI) }\end{array}$ \\
\hline Increased aortic stiffness (cf-PWV $>10 \mathrm{~m} / \mathrm{s})$ & $2.78(1.37,5.56)^{* *}$ & $1.69(0.94,3.13)^{\dagger}$ \\
Nephropathy & $2.27(1.16,4.35)^{*}$ & $1.75(0.92,3.33)^{\dagger}$ \\
Body height $(10 \mathrm{~cm}$ increment) & $1.25(0.79,1.98)$ & $1.45(0.98,2.14)^{\dagger}$ \\
Mean first-year HbA $1 \mathrm{cc}(1 \%$ increment) & $1.04(0.85,1.28)$ & $1.19(1.00,1.42)^{*}$ \\
Arterial hypertension & $6.67(0.90,49.9)^{\dagger}$ & $1.37(0.49,3.85)$ \\
\hline
\end{tabular}

Both analyses were further adjusted for the same covariates as in Table 3

${ }^{\dagger} p<0.10,{ }^{*} p<0.05,{ }^{* *} p<0.01$ 
peripheral neuropathy endpoints. The Bypass Angioplasty Revascularization Investigation 2 Diabetes (BARI 2D) cohort [11] did not find any difference in prevalence of DPN between the insulin-sensitising treatment (which provided better glycaemic control) and the insulin-providing treatment during the follow-up, although the insulin-sensitising group had a lower incidence of new DPN. In addition, the Danish arm of the Anglo-Danish-Dutch Study of Intensive Treatment in People With Screen-Detected Diabetes in Primary Care (ADDITION) [10], the Steno-2 Study [29] and the Veterans Affairs Diabetes Trial (VADT) [30] did not report any benefit of intensive glucose control treatment on the development of DPN compared with conventional treatment. A systematic review and meta-analysis in 2012 [12] confirmed the strong beneficial effect of enhanced glycaemic control on the prevention of DPN in patients with type 1 diabetes, with an annualised risk difference of $-1.84 \%(p<0.001)$, although it showed a weaker borderline significant effect (risk difference $-0.58 \% ; p=0.055)$ in patients with type 2 diabetes. Previous observational cohorts [31-33] have, however, shown strong associations between baseline $\mathrm{HbA}_{1 \mathrm{c}}$ levels and the future development of DPN.

Our results corroborate these previous studies by showing that, after a longer median follow-up of 6.2 years, the mean $\mathrm{HbA}_{1 \mathrm{c}}$ level during the first year of follow-up was shown to be a predictor of the future development/progression of DPN. This observation has an important clinical message: good glycaemic control should be established as soon as possible, ideally during the first year of follow-up, in order to prevent the development/progression of DPN.

The other independent predictors of the development/ progression of DPN have been reported in previous epidemiological studies involving patients with type 1 or type 2 diabetes $[8,27,31,32]$; this is particularly true of the presence of arterial hypertension $[8,27]$, a modifiable vascular risk factor. It is possible that the treatment of hypertension, as well as of other cardiovascular risk factors, could interfere with the development of peripheral arterial disease [34], an important contributor to classic diabetic foot ulceration.

There are, however, potential limitations to the interpretation of this study's results. First, the time interval between the baseline and final DPN evaluation of the participants was variable, ranging from 2 up to 10 years. However, there were no differences in the follow-up time between the patients with and without a development/progression of DPN (median 73 vs 75 months, respectively), and the multivariate regression analyses were further adjusted for the time interval between the two DPN assessments.

Second, this study began in 2004, so we used for the diagnosis of DPN the most commonly employed criterion, which was a minimum of two abnormalities in symptoms and signs, that is, clinically evident DPN, which corresponds to probable diabetic sensorimotor polyneuropathy in the more recent definition [1]. The current standard definition of DPN remains challenging as it combines clinical and electrophysiological criteria [1]. Nonetheless, other longitudinal studies investigating the development/progression of diabetic neuropathy have also not performed nerve conduction examinations for diagnosis [8-11, 30, 32, 33].

Third, assessing clinical DPN involves measures that are prone to a varying extent of subjectivity or observer bias. However, these measurement inaccuracies would actually underestimate the statistical significance towards the null hypothesis for the associations between risk factors and the development/progression of DPN. Finally, this study enrolled middle-aged to elderly high-risk individuals with longstanding type 2 diabetes. Therefore, our findings may not be generalisable to younger patients with recent-onset diabetes. Furthermore, this was an observational cohort so neither cause-and-effect relationships nor physiopathological mechanisms can be inferred, but only speculated.

In conclusion, this study demonstrated that increased aortic stiffness at baseline predicted the future development or progression of DPN, independent of metabolic control, suggesting a physiopathological link between macrovascular and microvascular abnormalities in type 2 diabetes. Prospective future studies with intensive multifactorial management, including the optimal control of metabolic and cardiovascular risk factors, possibly accompanied by a reduction in aortic stiffness, should address whether these interventions would be able to prevent or delay the development of DPN in patients with type 2 diabetes.

Funding This study was supported by grants from the Conselho Nacional de Desenvolvimento Científico e Tecnológico (CNPq, Brazil) and from the Fundação Carlos Chagas Filho de Amparo a Pesquisa do Estado do Rio de Janeiro (FAPERJ, Brazil).

Duality of interest The authors declare that there is no duality of interest associated with this manuscript.

Contribution statement CRLC drafted the manuscript, CBMM performed all the peripheral neuropathy assessments, and MTF performed all the cf-PWV measurements. All authors were involved in the patient follow-up and obtained the data. CRLC and GFS conceived and designed the study, analysed the data and are the guarantors of this study. All authors helped to interpret the results, reviewed the manuscript and approved the final version. CRLC and GFS had full access to all the data and take responsibility for the integrity of the data and the accuracy of data analysis.

\section{References}

1. Tesfaye S, Boulton AJ, Dyck PJ et al (2010) Diabetic neuropathies: update on definitions, diagnostic criteria, estimation of severity, and treatments. Diabetes Care 33:2285-2293 
2. Cameron NE, Eaton SE, Cotter MA, Tesfaye S (2001) Vascular factors and metabolic interactions in the pathogenesis of diabetic neuropathy. Diabetologia 44:1973-1988

3. Giannini C, Dyck PJ (1994) Ultrastructural morphometric abnormalities of sural nerve endoneurial microvessels in diabetes mellitus. Ann Neurol 36:408-415

4. Coppini DV, Bowtell PA, Weng C, Young PJ, Sönksen PH (2000) Showing neuropathy is related to increased mortality in diabetic patients - a survival analysis using an accelerated failure time model. J Clin Epidemiol 53:519-523

5. Boulton AJ, Vileikyte L, Ragnarson-Tennvall G, Apelqvist J (2005) The global burden of diabetic foot disease. Lancet 366:1719-1724

6. Gordois A, Scuffham P, Shearer A, Oglesby A, Tobian JA (2003) The health care costs of diabetic peripheral neuropathy in the US. Diabetes Care 26:1790-1795

7. Albers JW, Herman WH, Pop-Busui R, Diabetes Control and Complications Trial/Epidemiology of Diabetes Interventions and Complications Research Group et al (2010) Effect of prior intensive insulin treatment during the Diabetes Control and Complications Trial (DCCT) on peripheral neuropathy in type 1 diabetes during the Epidemiology of Diabetes Interventions and Complications (EDIC) Study. Diabetes Care 33:1090-1096

8. Tesfaye S, Chaturvedi N, Eaton SE, EURODIAB Prospective Complications Study Group et al (2005) Vascular risk factors and diabetic neuropathy. N Engl J Med 352:341-350

9. Ismail-Beigi F, Craven T, Banerji MA, ACCORD trial group et al (2010) Effect of intensive treatment of hyperglycaemia on microvascular outcomes in type 2 diabetes: an analysis of the ACCORD randomised trial. Lancet 376:419-430

10. Charles M, Ejskjaer N, Witte DR, Borch-Johnsen K, Lauritzen T, Sandbaek A (2011) Prevalence of neuropathy and peripheral arterial disease and the impact of treatment in people with screendetected type 2 diabetes: the ADDITION-Denmark study. Diabetes Care 34:2244-2249

11. Pop-Busui R, Lu J, Brooks MM, BARI 2D Study Group et al (2013) Impact of glycemic control strategies on the progression of diabetic peripheral neuropathy in the Bypass Angioplasty Revascularization Investigation 2 Diabetes (BARI 2D) Cohort. Diabetes Care 36:3208-3215

12. Callaghan BC, Little AA, Feldman EL, Hughes RA (2012) Enhanced glucose control for preventing and treating diabetic neuropathy. Cochrane Database Syst Rev, Issue 6, CD007543. doi:10. 1002/14651858.CD007543.pub2

13. Cavalcante JL, Lima JA, Redheuil A, Al-Mallah MH (2011) Aortic stiffness: current understanding and future directions. J Am Coll Cardiol 57:1511-1522

14. Kimoto E, Shoji T, Shinohara K et al (2003) Preferential stiffening of central over peripheral arteries in type 2 diabetes. Diabetes 52 : $448-452$

15. Cardoso CR, Ferreira MT, Leite NC, Barros PN, Conte PH, Salles GF (2009) Microvascular degenerative complications are associated with increased aortic stiffness in type 2 diabetic patients. Atherosclerosis 205:472-476

16. Kim ES, Moon SD, Kim HS et al (2011) Diabetic peripheral neuropathy is associated with increased arterial stiffness without changes in carotid intima-media thickness in type 2 diabetes. Diabetes Care 34:1403-1405

17. Laurent S, Cockcroft J, van Bortel L et al (2006) Expert consensus document on arterial stiffness: methodological issues and clinical applications. Eur Heart J 27:2588-2605

18. Cardoso CR, Ferreira MT, Leite NC, Salles GF (2013) Prognostic impact of aortic stiffness in high-risk type 2 diabetic patients: the
Rio de Janeiro Type 2 Diabetes Cohort Study. Diabetes Care 36: 3772-3778

19. Cardoso CR, Leite NC, Ferreira MT, Salles GF (2015) Prognostic importance of baseline and serial glycated hemoglobin levels in high-risk patients with type 2 diabetes: the Rio de Janeiro Type 2 Diabetes Cohort Study. Acta Diabetol 52:21-29

20. Salles GF, Leite NC, Pereira BB, Nascimento EM, Cardoso CR (2013) Prognostic impact of clinic and ambulatory blood pressure components in high-risk type 2 diabetic patients: the Rio de Janeiro Type 2 Diabetes Cohort Study. J Hypertens 31:2176-2186

21. Van Bortel LM, Laurent S, Boutouyrie P, Artery Society; European Society of Hypertension Working Group on Vascular Structure and Function; European Network for Noninvasive Investigation of Large Arteries et al (2012) Expert consensus document on the measurement of aortic stiffness in daily practice using carotid-femoral pulse wave velocity. J Hypertens 30:445-448

22. Young MJ, Boulton AJ, MacLeod AF, Williams DR, Sonksen PH (1993) A multicentre study of the prevalence of diabetic peripheral neuropathy in the United Kingdom hospital clinic population. Diabetologia 36:150-154

23. Symons MJ, Moore DT (2002) Hazard rate ratio and prospective epidemiological studies. J Clin Epidemiol 55:893-899

24. Yokoyama H, Yokota Y, Tada J, Kanno S (2007) Diabetic neuropathy is closely associated with arterial stiffening and thickness in type 2 diabetes. Diabet Med 24:1329-1335

25. Laurent $S$, Boutouyrie $P(2007)$ Recent advances in arterial stiffness and wave reflection in human hypertension. Hypertension 49: $1202-1206$

26. Ferreira MT, Leite NC, Cardoso CR, Salles GF (2015) Correlates of aortic stiffness progression in patients with type 2 diabetes: importance of glycemic control. The Rio de Janeiro type 2 diabetes cohort study. Diabetes Care 38:897-904

27. Forrest KY, Maser RE, Pambianco G, Becker DJ, Orchard TJ (1997) Hypertension as a risk factor for diabetic neuropathy: a prospective study. Diabetes 46:665-670

28. Martin CL, Albers JW, Pop-Busui R, DCCT/EDIC Research Group (2014) Neuropathy and related findings in the diabetes control and complications trial/epidemiology of diabetes interventions and complications study. Diabetes Care 37:31-38

29. Gaede P, Vedel P, Larsen N, Jensen GV, Parving HH, Pedersen O (2003) Multifactorial intervention and cardiovascular disease in patients with type 2 diabetes. N Engl J Med 348:383-393

30. Duckworth W, Abraira C, Moritz T, Investigators VADT et al (2009) Glucose control and vascular complications in veterans with type 2 diabetes. N Engl J Med 360:129-139

31. Partanen J, Niskanen L, Lehtinen J, Mervaala E, Siitonen O, Uusitupa M (1995) Natural history of peripheral neuropathy in patients with non-insulin-dependent diabetes mellitus. N Engl J Med 333:89-94

32. Adler AI, Boyko EJ, Ahroni JH, Stensel V, Forsberg RC, Smith DG (1997) Risk factors for diabetic peripheral sensory neuropathy. Results of the Seattle Prospective Diabetic Foot Study. Diabetes Care 20:1162-1167

33. van de Poll-Franse LV, Valk GD, Renders CM, Heine RJ, van Eijk JT (2002) Longitudinal assessment of the development of diabetic polyneuropathy and associated risk factors. Diabet Med 19: 771-776

34. Adler AI, Stevens RJ, Neil A, Stratton IM, Boulton AJ, Holman RR (2002) UKPDS 59: hyperglycemia and other potentially modifiable risk factors for peripheral vascular disease in type 2 diabetes. Diabetes Care 25:894-899 